Escritos Contables y de Administración, ISSN 1853-2063, vol. 4, n 1, 2013, págs. 81 a 107.

\title{
CONCIENCIA Y ACCIONES DE CONSUMO RESPONSABLE EN LOS JÓVENES UNIVERSITARIOS ${ }^{1}$ AWARENESS AND RESPONSIBLE CONSUMPTION IN UNIVERSITY YOUTHS
}

\author{
Enrique Carlos Bianchi², Belén Carmelé3, \\ Darío Tubaro, Juan Manuel Bruno ${ }^{5}$
}

Fecha de recepción: $17 / 03 / 2014$

Fecha de aceptación: 02/06/2014

\section{RESUMEN}

El presente trabajo se enmarca dentro de los Estudios sobre Consumo Responsable iniciados en forma conjunta por tres universidades nacionales en 2011: UNER-UNL-UNC.

Tiene por objetivo medir el nivel de conciencia, compromiso y de acción de las generaciones más jóvenes respecto del consumo de agua, energía, reciclaje, donaciones y de la compra en general. En este sentido, el trabajo se

\footnotetext{
${ }^{1}$ En el marco del proyecto RED EDUCA-AL de CONSUMO RESPONSABLE junto con las Universidad Nacional de Entre Ríos y la Universidad Nacional del Litoral. Proyecto SecytUNC 2012-2013.

2 Cátedra de Comercialización. Departamento de Administración. Facultad de Ciencias Económicas - Universidad Nacional de Córdoba (UNC), geomarket@arnet.com.ar.

3 Cátedra de Comercialización. Departamento de Administración. Facultad de Ciencias Económicas - UNC, belencarmele@gmail.com.

4 Cátedra de Comercialización. Departamento de Administración. Facultad de Ciencias Económicas - UNC, dariotubaro@hotmail.com.

5 Cátedra de Comercialización. Departamento de Administración. Facultad de Ciencias Económicas - UNC, jmbruno.mkt@gmail.com.
} 
centra en el componente conativo de la actitud del segmento estudiado, considerando los diferentes perfiles de jóvenes universitarios, poniendo en evidencia determinadas contradicciones entre el decir y el hacer.

El estudio cualitativo consistió en dinámicas grupales utilizando la técnica de collage para profundizar conceptos sobre consumo responsable, sociedad y ecología. En el cuantitativo, por medio de un cuestionario semiestructurado y a partir de una muestra de más de 400 casos de estudiantes universitarios de ambos sexo, se indagó sobre los estilos de vida de los jóvenes y también sobre los estilos de conductas responsables y sus acciones de consumo.

Los hallazgos del estudio muestran que los jóvenes lejos de hacer la revolución para transformar la sociedad, van forjando valores y conductas más proambiente fruto de la educación recibida. Las decisiones de consumo no son siempre racionales y conscientes, con diferencias de comportamientos según los estilos de vida donde los grupos más acorde con un estilo de consumo responsable son las jóvenes "Equilibradas" y "Workaholic" y de los jóvenes "Activistas" y "Luchadores".

Palabras claves: Consumo Responsable y Sustentable -Estilo de Jóvenes Ética del consumidor

\section{Abstract}

This paper is part of the Studies on Responsible Consumption initiated jointly by three national universities in 2011 - UNER-UNL-UNC.

It aims to measure the level of awareness, commitment and action of the younger generations as regards water consumption, energy, recycling, donations and purchase in general. In this sense, the study focuses on the conative component of attitude in the segment under analysis, considering the different profiles of university students, and highlighting certain contradictions between saying and doing.

The qualitative study consisted of group dynamics using the technique of collage to deepen concepts of responsible consumption, society and ecology. Whereas in the quantitative one, using a semi-structured questionnaire, a 
sample of more than 400 male and female university students were inquired not only about their lifestyles, but also about their style of responsible behavior and consumption actions.

Findings show that far from making a revolution to transform society, younger people are forging pro-environment values and behaviors as a result of the education received. It is also concluded that consumption decisions are not always rational and conscious, with differences in behavior according to lifestyles in which those befitting a responsible consumer style are the "Balanced" and "Workaholic" young females and the "Activist" and "Fighter" young males.

Key Words: Responsible and Sustainable Consumption - Youth Styles Consumer Ethics. 


\section{INTRODUCCIÓN}

El presente trabajo se enmarca dentro de los Estudios sobre Consumo Responsable iniciados en forma conjunta por tres universidades nacionales en 2011.

Tiene por objetivo medir el nivel de conciencia, compromiso y de acción de las generaciones más jóvenes respecto del consumo de agua, energía, reciclaje, donaciones y en la compra en general.

En este sentido, el trabajo se centra en el componente conativo de la actitud del segmento estudiado, considerando los diferentes perfiles de jóvenes universitarios, poniendo en evidencia determinadas contradicciones entre el decir y el hacer.

El objetivo de este estudio es en primer lugar, conocer los valores y actitudes de los jóvenes universitarios respecto del Consumo Responsable (CR);segundo, poder caracterizar los distintos perfiles clúster y por último, medir comportamientos concretos respecto de consumo responsable. Sobre este tema sostiene Moltedo Perfetti (2007: 204):

...mucho se ha dialogado sobre la responsabilidad social de las empresas, pero pocas veces se ha hablado de la responsabilidad social del consumidor...Un consumo responsable se centra en un consumo ético y solidario, y no en un consumo por el consumo.

Los primeros estudios fueron realizados en el año 2011 y expuestos en la XXV Educa-Al San Juan, permitieron tener una aproximación acerca del grado de información y concientización que existe en nuestra sociedad respecto al consumo y la autopercepción de las personas en cuanto a su rol de consumidores.

A partir de las presentaciones que se fueron efectuando en el marco del proyecto, surgió la necesidad de precisar las tipologías que surgen a partir de la autopercepción de las personas y contrastarla con las acciones efectivamente realizadas.

\section{MARCO TEÓRICO CONCEPTUAL}

Un primer abordaje a los temas de consumo, consumismo y consumo responsable, temas que conforman nuestro marco teórico fue presentado en la XXV EDUCA-AL. Se presentan seguidamente algunos extractos del mismo 
que permiten ilustrar sintéticamente los temas trabajados en dicha oportunidad (Almirón, Z. et al., 2011).

\subsection{REVISIÓN DE LA LITERATURA}

Puede caracterizarse el momento actual como la "era del consumo" porque en la sociedad, este ha aumentado en forma vertiginosa. En las sociedades no se trata de consumir bienes básicos y necesarios, sino que lo característico es el consumo de bienes superfluos. Se habla por ello de una sociedad consumista. Pero hablar de una sociedad consumista no es lo mismo que hablar de una sociedad en la que todo el mundo consume, porque es lógico y evidente que todo el mundo tiene que consumir siempre algo para poder sobrevivir. Adela Cortina (2003:24) la caracteriza como:

Una sociedad en la que se consume no lo necesario para la vida sino lo superfluo, y en la que además el consumo legitima la política y legitima la economía. La política se legitima si los políticos son capaces de conseguir un crecimiento económico ... mientras que la economía se legitima si cada vez se fabrican productos más sofisticados.

Sobre esta era reflexiona Reisch (2001) cuando afirma:

En la sociedad de consumo postmoderna el consumo ha perdido casi por completo su simple función de satisfacción de necesidades. En la actualidad desempeña funciones simbólicas como la autorrealización, la construcción de identidad, el control del estatus, el manejo de impresiones o el hedonismo imaginativo, entre otras. Además, ir de compras es una actividad de entretenimiento, ocio, compensación y evasión socialmente aceptada.

Muchos son los estudios referidos al tema del Consumo Responsable y las actitudes hacia el consumo. Son investigados por universidades y organismos públicos que describen el fenómeno del consumismo y del consumo ético y responsable (Aranque Padilla, 2003; Carrero Bosch et al., 2010; Ballesteros, 2011; Cortes Funes, 2011; Díaz, 2011). Ejemplo de ello, es el estudio realizado por Salcedo Arnal, A. y Garcés Prieto, J. (2006) sobre la relación entre ansiedad y consumo, cuyo antecedente es otro desarrollado 
desde 1998 a 2006, entre jóvenes de distintas regiones europeas. Dicho estudio concluyó, entre otras cuestiones que:

1) Los jóvenes tienen un nivel mucho más alto de adicción a los estímulos de compra, y un nivel más bajo de autocontrol económico que los adultos.

2) La mayoría de los jóvenes consumidores europeos tienen una importante tendencia consumista y de adicción a la compra, siendo la proporción de jóvenes "adictos al consumo" mucho mayor que la de adultos. Asimismo los autores afirman que las causas de esta adicción al consumo en los jóvenes son las siguientes:

a) Preexistencia de unos rasgos psicológicos específicos que combinan el ser "caprichoso" con la impulsividad, la labilidad y un nivel alto de ansiedad en relación con la compra.

b) La insatisfacción personal, la sensación de tedio o aburrimiento y la falta de alicientes no consumistas.

c) La mayor aceptación de valores consumistas y la vulnerabilidad psicológica hacia los mensajes que relacionan el consumo con la felicidad, el éxito social y el prestigio personal.

d) La atracción por los estímulos de consumo, como pasar el tiempo en hipermercados y grandes centros comerciales, ver anuncios, mirar escaparates, entrar en comercios, aunque solo sea a mirar, etc. Son actividades que por sí mismas no pueden considerarse consumo, pero que en los jóvenes provocan un efecto muy intenso de incitación a la compra y predisponen a la adicción al consumo.

e) El bajo nivel de autoestima y la desconfianza hacia las propias aptitudes o habilidades.

Por último, dicho estudio destaca, entre otras conclusiones, que un nivel alto de ansiedad está claramente correlacionado con la insatisfacción personal, la sensación de tedio o aburrimiento y la falta de alicientes no consumistas. La frecuente conjunción de todos estos factores en las mismas personas, explica los problemas de adicción al consumo, la atracción desmedida por los estímulos consumistas, la falta de autocontrol en el gasto y la compra no responsable, que son problemas de gran importancia y rápido crecimiento en la sociedad actual con especial incidencia en los jóvenes. 
Para Cortina, A. (2002 y 2003), el consumo, como fenómeno social, tiene consecuencias dentro y fuera de la esfera individual y, por tanto, es también una oportunidad de justicia si los imperativos éticos participan en el comportamiento. Aunque, todavía el consumo de masas sigue siendo el gran nicho de la demanda y que sus valores y referencias siguen todavía mayoritariamente vigentes, en algunos casos desgastados y degradados por el abuso de un modelo con evidentes signos de colapso (exceso de publicidad, atascos, disfunciones de las aglomeraciones, pérdida de identidad, etc.), sostiene Alonso (2006).

La idea de un consumidor socializado - ni dominado, ni racional puroque elige en espacios de decisión limitados, pero existentes. Nos encontramos con una mezcla realista de manipulación y libertad de compra, de impulso y reflexión, de comportamiento condicionado y uso social de los objetos y símbolos de la sociedad de consumo. En este sentido, desempeña funciones simbólicas como la autorrealización, la construcción de identidad, el control del estatus, el manejo de impresiones o el hedonismo imaginativo, entre otras. (Reisch, 2001).

Por otra parte, sabemos que los jóvenes son muy susceptibles a los fenómenos de grupos, de modas y que pueden ser presa fácil del consumo de la sociedad postcapitalista, donde la publicidad sirve de cebo (Hayes, 1961 y 1978), donde roles, ritos y estereotipos compartidos dominan nuestra cultura actual (Martínez, 2008). Autores como Braudrillard (1970) afirman que el consumo ha pasado a ser el aspecto más importante de la acumulación capitalista, donde los objetos que se consumen proceden de representaciones, sueños y fantasías donde se han introducido artificialmente los individuos, hoy fuertemente potenciado por las nuevas tecnologías, como: youtube, facebook, el acceso a la televisión por cable, entre otras.

Diferentes son las perspectivas teóricas respecto del consumo - desde las utilitaristas, las relacionales y las del realismo crítico- y las posturas ideológicas sobre el comportamiento en relación al consumo - neoliberal, dependencia, poder del ciudadano, ciudadanía del consumidor, entre otras - que han sido abordadas en el trabajo de Almirón et al. (2011) del cual queremos rescatar las siguientes ideas o hipótesis, que deseamos verificar por el presente estudio: 
- Si bien existe una soberanía del consumidor donde este elige o vota (Hayes, 1961 y 1978), las decisiones de consumo no son racionales, sino que muchas son impulsivas o emocionales.

- Los procesos de estratificación social y las tecnologías tienden a un mayor aislamiento social, actitud propia de los adolescentes y de los jóvenes.

- Los jóvenes no desean hacer la revolución para transformar la sociedad como quisiera Miller (1999), sino disfrutar de los beneficios de un estado de bienestar forjado por otros y de las ventajas de las tecnologías y medios de comunicación.

- El compromiso con los otros para generar el cambio social es bastante bajo, pues el consumidor o esta alienado o es pasivo (Baudrillard, 1974; Bell, 1978; Jameson, 1999;Bauman, 2002).

\subsection{CONSUMO RESPONSABLE}

Según Iglesias (2009) el concepto de consumo responsable es muy amplio, como lo es la propia actividad de consumir, pero puede sintetizarse en tres ejes:

- Un Consumo Ético (basado en valores, deliberado, consciente): en el que se introduzcan valores como una variante importante a la hora de consumir o de optar por un producto. Con especial énfasis en la austeridad como un valor en relación con la reducción para un consumo ecológico, pero también frente al crecimiento económico desenfrenado y al consumismo, como forma de alcanzar el bienestar y la felicidad.

- Un Consumo Ecológico (3R's), que incluye, las famosas "erres" del movimiento ecologista: Reducir, Reutilizar y Reciclar, pero en el que también se incluyen elementos tan imprescindibles como la agricultura y ganadería ecológicas, la opción por la producción artesanal, entre otros.

- Un Consumo Social o Solidario (vinculado a las relaciones sociales, laborales y RSC), en el que entraría también el Comercio Justo, es decir, el consumo en lo que se refiere a las relaciones sociales y 
condiciones laborales en las que se ha elaborado un producto o prestado un servicio. Se trata de pagar lo justo por el trabajo realizado, tanto a gente de otros países como a las más cercanas, en nuestro ámbito local; se trata de eliminar la discriminación, ya sea a causa del color de la piel o por diferente origen, o por razón de género o religión; se trata de potenciar alternativas sociales y de integración y de procurar un nuevo orden económico internacional.

Es decir, que entendemos el Consumo Responsable como una actitud. Según Alonso Rivas y Grande Esteban (2012:351) "la actitud constituye una creencia o sentimiento aprendido que predispone a una persona a reaccionar de un modo determinado ante un objeto, persona o situación". Una actitud está formada por tres componentes básicos:

- El componente cognoscitivo: agrupa el conjunto de conocimientos, creencias y asociaciones hacia un objeto determinado.

- El componente afectivo: resume los sentimientos positivos y negativos, así como las emociones del individuo.

- El componente del comportamiento: se refiere a las intenciones de comportamiento, representa la tendencia a la acción.

Las actitudes no son inamovibles, sino que las empresas pueden influir sobre los consumidores si logran modificar alguno de sus tres componentes. Este hecho resulta de suma importancia cuando se pretende que el público objetivo mantenga o mejore actitudes positivas hacia sus productos $\mathrm{o}$ marcas, o para este caso en particular, hacia el consumo responsable.

Para Alonso Rivas y Grande, Esteban (2012), sobre el componente cognoscitivo se puede actuar brindando nueva información sobre los atributos del objeto en estudio; sobre el componente afectivo, mediante experiencias agradables proporcionadas por los estímulos comerciales a través de música, imágenes, humor, etc.

Por último, la influencia sobre el componente del comportamiento es factible de lograr realizando acciones en puntos de contacto con los consumidores: organización de eventos, participación en actividades de concientización, cuidado del medioambiente, etc. Estas acciones tienden a favorecer un mejor aprendizaje y reforzar la actitud positiva buscada. 


\section{Metodología de estudio}

El presente trabajo se desarrolla a partir de una investigación cualicuantitativa. El estudio cualitativo consistió en dinámicas grupales de hombres y mujeres de entre 20-35 años, utilizando la técnica de collage para profundizar los conceptos sobre sociedad, consumo, responsabilidad social empresaria y ecología. (Martínez, 2008)

En el estudio cuantitativo se trabajó a partir de la revisión de dos encuestas anteriores: Encuesta de Estilo de Vida de los Jóvenes Universitarios (Bianchi, 2007 y 2008) y de Consumo Responsable (Almirón et al., 2011). Se revisaron los ítems, ampliaron, redujeron y modificaron a los fines de poder, a partir de ellos, conformar los clúster de estilo de vida y de Consumo Responsable;se adicionaron ítems para medir acciones concretas de Consumo Responsable en temáticas tales como: agua, energía, reciclado domiciliarios, donaciones y proceso de evaluación de compra en general. Puede consultar los ítems utilizados en los anexos I y II.

Se diseñó una muestra no probabilística por cuota (sexo, edad, facultad) de 422 casos. El relevamiento fue el mes de octubre de 2011. Se procesaron los datos con DYANE versión 4, efectuándose análisis univariados, bivariados y multivariados.

La ficha técnica se puede apreciar en la tabla 1.

Tabla 1. Ficha Técnica de la investigación

\begin{tabular}{|c|c|}
\hline Naturaleza metodológica & Cuantitativa Exploratoria. \\
\hline Técnica metodológica & $\begin{array}{l}\text { Encuesta con cuestionario semi-estructurado, en forma } \\
\text { personal. }\end{array}$ \\
\hline Universo & Jóvenes universitarios de la ciudad de Córdoba. \\
\hline Elementos de muestreo & $\begin{array}{l}\text { Jóvenes universitarios de la Universidad Nacional de } \\
\text { Córdoba. }\end{array}$ \\
\hline Unidades de muestreo & Personas. \\
\hline Ámbito geográfico & Ciudad de Córdoba. \\
\hline Tamaño de la muestra & 418 casos válidos. \\
\hline Procedimiento de muestreo & $\begin{array}{l}\text { Muestreo no probabilístico: } \\
\text { - Control por cuota de sexo y edad. } \\
\text { - Control por facultad de pertenencia. }\end{array}$ \\
\hline Período de recolección & Del 3al 17 de octubre de 2012. \\
\hline Ejecución del trabajo de campo & Cátedra de Comercialización 2 - FCE - UNC. \\
\hline
\end{tabular}


Se presentan las siguientes tipologías:

- Ocho clústers de estilo de vida de los jóvenes:

- Ellas: Barbies-Workaholic-Intelectuales y Equilibradas.

- Ellos: Equilibrados - Luchadores - Solidarios y Activistas.

- Cuatro clústers de Consumo Responsable:

- Anticonsumistas - Indiferentes Apáticos - Shopper Compulsivos y Consumidores Responsables.

La tabla 2 muestra el perfil de la muestra seleccionada y la tabla 3, las tipologías encontradas, el perfil de los jóvenes universitarios y su perfil como comprador responsable.

Tabla 2. Perfil de la muestra de Jóvenes Universitarios

\begin{tabular}{|c|c|c|}
\hline & Casos & $\%$ \\
\hline \multicolumn{3}{|l|}{ Sexo } \\
\hline Femenino & 259 & 38 \\
\hline Masculino & 159 & 62 \\
\hline \multicolumn{3}{|l|}{ Edad } \\
\hline 18 a 30 años & 376 & 90 \\
\hline Más de 31 años & 43 & 10 \\
\hline \multicolumn{3}{|l|}{ Facultad } \\
\hline Ciencias Médicas & 74 & 18 \\
\hline Ciencias Económicas & 57 & 14 \\
\hline Derecho y Ciencias Sociales & 46 & 11 \\
\hline Arquitectura, Urbanismo yDiseño & 40 & 10 \\
\hline Escuela de Trabajo Social & 40 & 10 \\
\hline Psicología & 33 & 8 \\
\hline Filosofía y Humanidades & 29 & 7 \\
\hline Lenguas & 26 & 6 \\
\hline Exactas, Físicas y Naturales & 25 & 6 \\
\hline Ciencias Agropecuarias & 16 & 4 \\
\hline Odontología & 15 & 4 \\
\hline Ciencias Químicas & 12 & 3 \\
\hline Matemática, Astronomía y Física & 6 & 1 \\
\hline
\end{tabular}


Enrique Carlos Bianchi, Belén Carmelé, Darío Tubaro, Juan Manuel Bruno

Tabla 3. Tipologías de Jóvenes Universitarios y de Comportamiento Responsable

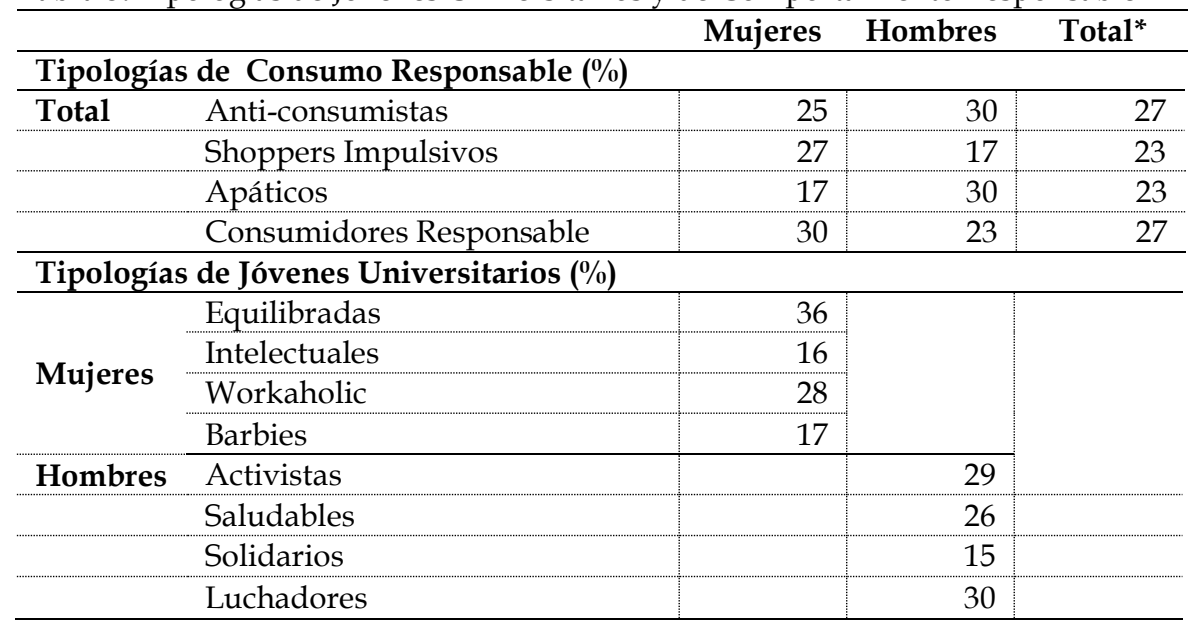

*ponderados mujeres (60 \%) y hombres (40\%).

Para una mayor comprensión de estas tipologías puede consultarse el Anexo III, donde encontrara una breve descripción de cada una de ellas.

\section{Principales Resultados}

La tipología de los jóvenes universitarios se generaron por separados hombres y mujeres; el perfil de comportamiento responsable se estimó sobre la muestra total. Primero, mostramos los resultados de las tipologías de los jóvenes y su perfil responsable y en segundo término, los hábitos o prácticas concretas (acciones) que estos realizan.

\subsection{TIPOLOGÍAS DE JÓVENES UNIVERSITARIOS Y SU COMPORTAMIENTO RESPONSABLE}

Los resultados de la investigación muestran los cruces entre los perfiles encontrados, véase tabla 3, y las acciones concretas realizadas en términos de consumo responsable. En la tabla 4 puede verse cada grupo de mujeres - equilibradas, Intelectuales, Worksholic y Barbies - y su perfil de consumidor responsable:

- Del grupo de las Equilibradas, la mayoría son Consumidores Responsables (41,5\%). 
- Del grupo de las Intelectuales la mayoría con una diferencia notable son Anti-consumistas (61 \%) a la hora de consumir.

- Las Workaholic son en su mayoría también Anti-consumistas (33\%), pero, con una proporción similar, hay muchas Consumidoras Responsables (30\%).

- Y en el grupo de las Barbies podemos ver que el consumo que predomina es el Shoppers compulsivo (58,8\%)

Tabla 4. Las mujeres universitarias y el consumo responsable

\begin{tabular}{|c|c|c|c|c|}
\hline \multicolumn{5}{|c|}{ Tipología de las mujeres universitarias y el consumo responsable } \\
\hline & $\begin{array}{c}\text { Anticonsumistas } \\
\text { Conscientes }\end{array}$ & $\begin{array}{l}\text { Shoppers } \\
\text { Impulsivos }\end{array}$ & Apáticos & $\begin{array}{c}\text { Consumidores } \\
\text { Responsables } \\
\end{array}$ \\
\hline \multicolumn{5}{|c|}{$\mathrm{n}=259$ (en $\%, 100 \%$ en fila $)$} \\
\hline Barbies & 9,8 & 58,8 & 11,8 & 19,6 \\
\hline Workaholic & 33,0 & 26,0 & 11,0 & 30,0 \\
\hline Intelectuales & 61,0 & 2,5 & 19,5 & 17,0 \\
\hline Equilibradas & 12,8 & 20,2 & 25,5 & 41,5 \\
\hline Total & 25 & 27 & 17 & 30 \\
\hline
\end{tabular}

De los 159 entrevistados masculinos, se observa que el grupo de consumo responsable predominante, es el de los Anticonsumistas o Indiferentes.

- La mayoría de los Activistas son Consumidores Responsables (31 \%) a la hora de consumir productos.

- Los Saludables se quejan de las conductas no responsables, pero a la hora de actuar ellos mismos no lo son, es decir, sólo aparentan serlo, son Indiferentes apáticos (47,6 \%) y Shopper Compulsivos (31\%).

- Los Solidarios, la mayoría son Anti-consumistas (62,5\%).

- Los Luchadores se dividen entre Anti-consumistas (38,3\%), Indiferentes Apáticos (29,8 \%) y Consumidores Responsables (27,7 \%).

Tabla 5. Los hombres universitarios y el Consumo Responsable

Tipología de los hombres universitarios y el consumo responsable

\begin{tabular}{|c|c|c|c|c|}
\hline & $\begin{array}{l}\text { Anticonsumistas } \\
\text { Conscientes }\end{array}$ & $\begin{array}{l}\text { Shoppers } \\
\text { Impulsivos }\end{array}$ & Apáticos & $\begin{array}{l}\text { Consumidores } \\
\text { Responsables }\end{array}$ \\
\hline \multicolumn{5}{|c|}{$n=159($ en $\%, 100 \%$ en fila $)$} \\
\hline Luchadores & 38,3 & 4,3 & 29,8 & 27,7 \\
\hline Solidarios & 62,5 & 4,2 & 20,8 & 12,5 \\
\hline Saludables & 7,1 & 31,0 & 47,6 & 14,3 \\
\hline Activistas & 23,9 & 23,9 & 21,7 & 31,0 \\
\hline Total & 30 & 17 & 30 & 23 \\
\hline
\end{tabular}


Del estudio surge que hay diferencias en el perfil de Consumo Responsable agrupados por disciplinas de estudio, como muestra la tabla 6:

- Los de Ciencias Económicas, Exactas y Arquitectura destacan por un porcentaje menor de Shopper Compulsivos (14\%) y un tercio se define como Consumidores Responsables (35\%).

- Los de Lenguas, Filosofía y Psicología son los que presentan un mayor caso de pertenecientes al grupo de los Anticonsumistas (38,9\%), seguidos de los de Derecho y Trabajo Social. Además, los de Lenguas, Filosofía y Psicología también destacan por un alto porcentaje de Consumidores Responsables (25,3\%).

- Por lo que respecta a los de Ciencias médicas, Química, naturales y Odontología predominan grupos bien diferentes: los Shoppers Compulsivos (33,7 \%) Indiferentes Apáticos (25\%) y Consumidores Responsables (26,9\%).

Tabla 6. Consumo Responsable por Disciplina

\begin{tabular}{|c|c|c|c|c|}
\hline \multicolumn{5}{|c|}{ Consumo Responsable por disciplina } \\
\hline & $\begin{array}{c}\text { Anticonsumistas } \\
\text { conscientes }\end{array}$ & $\begin{array}{l}\text { Shoppers } \\
\text { Impulsivos }\end{array}$ & Apáticos & $\begin{array}{c}\text { Consumidores } \\
\text { Responsables }\end{array}$ \\
\hline \multicolumn{5}{|l|}{$n=418($ en $\%, 100$ en fila $)$} \\
\hline Derecho y Trabajo Social & 33,3 & 22,6 & 22,6 & 21,5 \\
\hline Lengua-Filosofía-Psicología & 38,9 & 15,8 & 20 & 25,3 \\
\hline $\begin{array}{l}\text { Médicas-Químicas- } \\
\text { Odontología }\end{array}$ & 14,4 & 33,7 & 25 & 26,9 \\
\hline $\begin{array}{l}\text { Cs.Es.-Exactas- } \\
\text { Arquitectura }\end{array}$ & 26,2 & 14,3 & 23,8 & 35,7 \\
\hline Total & 27 & 23 & 23 & 27 \\
\hline
\end{tabular}

\subsection{HÁbITOS DE CONSUMO Y ACCIONES RESPONSABLES}

Estas acciones se midieron en una escala de frecuencia del acto o de la práctica responsable de 5 puntos: $1=$ "nunca", 2="casi nunca", 3="ocasio-nalmente", $4=$ "casi siempre", $5=$ "siempre".

En el consumo de agua, en términos generales, tanto hombres como mujeres son poco conscientes de la escasez de agua y actúan medianamente de forma responsable. 
Tabla 7. Hábitos relacionados con el Cuidado del Agua

\begin{tabular}{l|r|r|r}
\hline \multicolumn{2}{l}{ Hábitos relacionados con el cuidado del Agua } \\
\hline & $\begin{array}{c}\text { Nunca - } \\
\text { Casi Nunca }\end{array}$ & Ocasional & $\begin{array}{c}\text { Casi siempre- } \\
\text { Siempre }\end{array}$ \\
\hline $\mathbf{n = 4 1 8}$ (en \%, 100 en fila) & 29,1 & 15,8 & 55,1 \\
\hline Cerrar el pico mientras se cepilla los dientes & 35,5 & 20,4 & 44,1 \\
\hline Lava el auto con un balde & 40,0 & 23,2 & 36,8 \\
\hline Regar las plantas con balde & 57,3 & 14,0 & 28,7 \\
\hline Lavar platos en un recipiente & 84,1 & 10,2 & 5,7 \\
\hline Cerrar la ducha mientras se enjabona & & & \\
\hline
\end{tabular}

Son las Consumidores Responsables y los Anticonsumistas los que destacan por el ahorro de agua. Las menos responsables son las Shopper Compulsivas y Barbies y las Indiferentes Apáticas en el caso de las Mujeres e Indiferentes Apáticos en el caso de los hombres.

Del estudio surge que la mayoría de los integrantes de cada grupo nunca cierra la ducha mientras se enjabona (78,72 \% de Indiferentes Apáticos, 65,18 \% de Consumidores Responsables, 57,89 \% de Shopper Compulsivos y un 42,86 \% de Anticonsumista.

Sin embargo, si lo hacen mientras se cepillan los dientes, resultando la mitad de los Anticonsumistas (59,82 \%) y Consumidores Responsables (50,89 \%) y en menor medida los Shopper Compulsivos (34,38\%) y los Indiferentes Apáticos (34,78 \%).

La mayoría de hombres y mujeres nunca lavan los platos en un recipiente y luego enjuagan. A pesar de esto, en cada grupo son menos de la mitad los que nunca lo hacen, pudiendo destacar que muchos lo realizan ocasionalmente. (En cada grupo rondan entre el 23 y $36 \%$ de los que nunca lo practican).

A la hora de lavar el auto o regar las plantas, la mayoría de los Anticonsumistas, Shopper Compulsivos y Consumidores Responsables, siempre utilizan un balde para ahorrar agua. Mientras que la mayoría de los Indiferentes Apáticos nunca lo realiza. Igualmente están bastante repartido entre los que nunca lo realizan, los que lo hacen ocasionalmente y siempre.

En el caso del consumo de energía, casi todos los encuestados desenchufan los artefactos eléctricos y apagan las luces cuando nadie las utiliza. 
Tabla 8. Hábitos relacionados con el ahorro de energía

\begin{tabular}{|c|c|c|c|}
\hline \multicolumn{4}{|l|}{ Hábitos relacionados con ahorro de energía } \\
\hline & $\begin{array}{c}\text { Nunca - } \\
\text { Casi Nunca }\end{array}$ & Ocasional & $\begin{array}{l}\text { Casi siempre- } \\
\text { Siempre }\end{array}$ \\
\hline \multicolumn{4}{|l|}{$n=418$ (en $\%, 100$ en fila) } \\
\hline Apagar luces encendidas cuando se retira & 10,1 & 6,3 & 83,5 \\
\hline $\begin{array}{l}\text { No dejar prendidos los calefactores/aires } \\
\text { acon. }\end{array}$ & 8,2 & 10,1 & 81,8 \\
\hline $\begin{array}{l}\text { Apagar las luces de los artefactos } \\
\text { electrónicos }\end{array}$ & 24,0 & 6,5 & 69,5 \\
\hline Desenchufar el cargador de celular & 23,4 & 11,4 & 65,2 \\
\hline
\end{tabular}

Hay una unanimidad casi absoluta en la práctica que más se hace para ahorrar energía: apagar calefactores o el aire acondicionado si no se necesitan. La mayor parte de Shopper Compulsivos "casi siempre" realiza lo primero, mientras que la mayoría de los demás grupos "siempre", destacándose Anticonsumistas con un 58 \%.Los Consumidores Responsables son las que más adoptan dichas buenas costumbres y los Shopper Impulsivos son los que menos consciencia de ahorro energético tienen.

Respecto de las acciones de reciclaje, la mayoría no suele separar sus residuos en sus hogares, pero si colaborar con reciclado de papel, pilas, hojas impresas y bolsas de supermercado.

Tabla 9. Hábitos relacionados con el reciclado

Hábitos relacionados con el reciclado

\begin{tabular}{l|rrr}
\hline & $\begin{array}{c}\text { Nunca- } \\
\text { Casi Nunca }\end{array}$ & Ocasional & $\begin{array}{c}\text { Casi siempre- } \\
\text { Siempre }\end{array}$ \\
\hline $\mathbf{n = 4 1 8}$ (en \%, 100 en fila) & \multicolumn{2}{c}{} & \\
\hline $\begin{array}{l}\text { Reutilizar las bolsas de nylon del } \\
\text { supermercado }\end{array}$ & 16,5 & 5,1 & 78,5 \\
\hline $\begin{array}{l}\text { Reutilizar las hojas impresas } \\
\text { Depositar las pilas en lugares específicos }\end{array}$ & 28,7 & 15,3 & 56,1 \\
\hline $\begin{array}{l}\text { Los productos de tecnología que descarto, } \\
\text { los dono o entrego para reciclar }\end{array}$ & 30,8 & 15,7 & 53,5 \\
\hline $\begin{array}{l}\text { Separo residuos (papel-plástico-vidrio) en } \\
\text { casa }\end{array}$ & 64,8 & 21,4 & 13,8 \\
\hline
\end{tabular}

La mayoría de los encuestados no suele separar sus residuos. Destacándose en mayor medida, el grupo de los Anticonsumistas con un $72,63 \%$ de dicho total y un 58 \% de los Consumidores Responsable. 
Todos acostumbran a reciclar las pilas menos el grupo de los Indiferentes Apáticos, el 33,68 \% (mayoría), nunca lo hace. Muy pocos acostumbran a donar los productos de tecnología antiguos para su reciclado.

La mayor parte de los grupos de los Anticonsumistas y los Consumidores Responsables siempre reutilizan las bolsas de supermercado, al igual que los demás grupos en menor medida. Sin embargo a la hora de reutilizar hojas impresas, el porcentaje de cada grupo es mucho menor, siendo el que más lo realiza el 55,75\% de los Anticonsumistas.

Casi ninguno de los entrevistados tiene en cuenta que los envases de sus productos son reciclables. Dentro de la minoría que sí lo hace, predominan los Anticonsumistas 35,24 \% de dicho total y los Shopper con un $32 \%$ de su total.

En cuanto a las acciones sociales de donación y colaboración con las Organizaciones de la Sociedad Civil (OSC), muy pocos acostumbran a donar los productos de tecnología antiguos para su reciclado o juntar tapitas, no así con la ropa usada.

Buena parte de los grupos de los Anticonsumistas y Consumidores Responsables (51,33 \% y 40,54\% respectivamente), dona ropa que no utiliza o la entrega para que sea reciclada.

La mayoría de los hombres y mujeres encuestados no colabora con OSC. Solo un 15,18 \% de los Anticonsumistas siempre lo hace. Sin embargo, a la hora de juntar tapitas muchos lo hacen aunque no es significativo el porcentaje, rondando entre el $12 \%$ y $19 \%$ de cada grupo.

Se necesita reforzar las campañas y la conciencia entre los estudiantes, de la gran contribución que significa para las Organizaciones de la Sociedad Civil (OSC), la recolección de tapitas (se realiza en la Facultad de Cs. Es.) y la recolección de papel.

Tabla 10. Hábitos relacionados la ayuda a OSC

\begin{tabular}{|c|c|c|c|}
\hline \multicolumn{4}{|l|}{ Hábitos relacionados con la ayuda a OSC } \\
\hline & $\begin{array}{c}\text { Nunca - } \\
\text { Casi Nunca }\end{array}$ & Ocasional & $\begin{array}{l}\text { Casi siempre } \\
\text { - Siempre }\end{array}$ \\
\hline \multicolumn{4}{|l|}{$\mathrm{n}=418$ (en $\%, 100$ en fila $)$} \\
\hline $\begin{array}{l}\text { La ropa que no uso la dono o entrego para } \\
\text { reciclar }\end{array}$ & 22,0 & 25,8 & 52,2 \\
\hline Recolecto tapitas de gaseosas para los OSC & 59,1 & 18,9 & 22,0 \\
\hline Donar papel a OSC que recaudan fondos & 67,9 & 18,2 & 13,8 \\
\hline Donar productos de tecnologías antiguos & 62,5 & 20,8 & 16,7 \\
\hline
\end{tabular}


Respecto de la conciencia en el acto de compra, la mitad tiene consciencia clara sobre la exigencia de la factura de compra y eligen productos y marcas originales. No prestan atención al tipo de envase, si daña o si es reciclable.

Tabla 11. Hábitos relacionados con el proceso de compra

\begin{tabular}{|c|c|c|c|}
\hline \multicolumn{4}{|c|}{ Hábitos relacionados con el proceso de compra } \\
\hline & $\begin{array}{c}\text { Nunca - } \\
\text { Casi Nunca }\end{array}$ & Ocasional & $\begin{array}{l}\text { Casi siempre } \\
\text { - Siempre }\end{array}$ \\
\hline \multicolumn{4}{|l|}{$\mathrm{n}=418$ (en $\%, 100$ en fila) } \\
\hline Entre original y copia, compro original & 22,8 & 27,2 & 50 \\
\hline Exijo la factura en los negocios & 35,5 & 23,2 & 41,3 \\
\hline $\begin{array}{l}\text { Leo las etiquetas cuando compro un } \\
\text { producto nuevo }\end{array}$ & 31,2 & 30,6 & 38,2 \\
\hline $\begin{array}{l}\text { Llevo mi propia bolsa / carrito al } \\
\text { supermercado }\end{array}$ & 71,6 & 12,3 & 16,1 \\
\hline $\begin{array}{l}\text { Me fijo que el envase sea reciclable y no } \\
\text { dañe el ambiente }\end{array}$ & 67,6 & 21,9 & 10,6 \\
\hline
\end{tabular}

Los grupos donde la mayor parte de los integrantes reclaman factura de compra son los Anticonsumistas y los Consumidores Responsables, aunque menos de la mitad. La mayoría de Shopper y los Indiferentes Apáticos lo realizan ocasionalmente.

La mayoría de los grupos valoran la marca de un producto si se les presenta una copia de menor costo. La mayor parte afirma que ocasionalmente si se les presenta dicha situación, optan por el original. Sin embargo, el grupo que aparenta ser más marquista son los Consumidores Responsables de los cuales el $25 \%$ lo haría casi siempre y el $21 \%$ siempre.

Dentro de los Consumidores Responsables, las mujeres demuestran llevar a cabo más acciones responsables que los hombres, los cuales destacan por su diversidad de opiniones.

Los Anticonsumistas son los que más leen las etiquetas a la hora de comprar un producto nuevo ( $42 \%$ de su total).

\section{DisCUSIÓN DE LOS RESUltados}

La investigación pone de manifiesto los diferentes estilos de vida, tanto de los hombres como de las mujeres universitarias, y su relación con el consumo responsable. Hemos descubierto que los perfiles de estilos de vida más proclives a una actitud que denominamos Consumo Responsable son: 
- En el caso de las mujeres universitarias, las "Equilibradas" y las "Workaholic" (las "Intelectuales" tienen un perfil anticonsumista), $\mathrm{y}$

- En los hombres universitarios, los "Activistas" y los "Luchadores".

Lo que se denota es que el Consumo Responsable como decíamos en la definición conceptual, se caracteriza por los siguientes rasgos (Carrero Bosch et al., 2010):

- Se trata de un consumo consciente y deliberado. El consumidor decide ponderando distintas variables, como precio, conveniencia y factores sociales. (Szmigin et al., 2009)

- Se realiza en forma rutinaria, siendo un hábito y no una compra puntual. Se considera consumidor responsable al que evalúa los efectos de sus decisiones de compra de forma rutinaria.

- Se actúa buscando el interés externo (centrado en otros) y no interno (centrado en sí mismo).

- Se busca, como fin último, modificar el contexto o las estructuras de mercado actuando sobre las prácticas empresariales o institucionales. (Micheletti, 2003).

Respecto de las hipótesis previas sobre la actitud de los jóvenes, podemos afirmar que existen tipologías diferentes que ponen en evidencia que si bien existe una soberanía del consumidor donde este elige o vota (Hayes, 1961 y 1978), las decisiones de consumo no son siempre racionales como en los "Consumidores Responsables" y "Anticonsumistas", ni siempre impulsivas o emocionales como en los "Shoppers" o más rutinarias como en los "Apáticos". Se comprueba que el compromiso con los otros para generar el cambio social es bastante bajo, así como el compromiso con las OSC en apoyar sus campañas de fondos y en sus acciones. Pues el consumidor o esta alienado como en el caso de los "Shoppers" o está pasivo como en el caso de los "Apáticos" (Baudrillard, 1974; Bell, 1978; Jameson, 1999; Bauman, 2002).

Por último, que los jóvenes no desean hacer la "revolución" para transformar la sociedad como quisiera Miller (1999), sino disfrutar de los beneficios de un estado de bienestar forjado por otros y de las ventajas de las tecnologías y medios de comunicación, depende de los perfiles y debe ser profundizado. 
Desde el punto de vista del comportamiento hay acciones pro Consumo Responsable que ya están siendo asumidas por los grupos de estudiantes, siendo las más relevantes las que sintetizamos a continuación:

- Ahorro de agua, en los casos de lavarse los dientes y lavar el auto.

- Ahorro de energía, cuando se trata de apagar luces, calefactores eléctricos, artefactos eléctricos y desenchufar celulares, etc.

- Reciclado, se reutilizan hojas de papel, se depositan pilas en lugares específicos.

- Donaciones de ropa usada a los más necesitados y

- Respecto del proceso de compra: pedir la factura, búsqueda del original

Las acciones que menos se asumen son:

- El reciclado en el hogar por no haber un sistema social y logístico que lo estimule y lo haga sencillo.

- Llevar la bolsa al supermercado para evitar usar plástico, por una pérdida de costumbre en las generaciones más jóvenes.

Existen diferentes enfoques para lograr el cambio de conducta desde el punto de vista del marketing social (Santesmases Mestre, et al., 2004); dichos enfoques pueden ser:

- Legal, por medio de prohibiciones.

- Tecnológico, dando nuevas soluciones que permitan hacer las cosas de manera diferente.

- Económico, por medio de sanciones y multas.

- Informativo, brindando información a los consumidores.

- Persuasivo, a través de campañas de concientización y persuasión.

Si bien en la presente investigación no se han relevado de manera formal las acciones de las empresas, gobiernos y OSC para lograr el cambio social hacia un consumo responsable y sustentable, nos atrevemos a afirmar hipotéticamente lo siguiente, como base para futuras investigaciones en estos campos específicos:

- El ahorro en materia de agua y energía se debe a un enfoque informativo y persuasivo pero fundamentalmente económico. El sistema tarifado de consumo, penaliza el mayor consumo y por otro, subsidia a los sectores de ingresos más bajos. 
- En las donaciones de ropa, papel, tapitas, etc. prima un enfoque persuasivo, con pocos incentivos económicos y algunos sociales (ayudar a otros a través de las OSC), por lo que los resultados son buenos, pero podrían ser mejores.

- Respecto del reciclado de basura en el hogar, la falencia está en el enfoque tecnológico (medios materiales y logístico) para facilitar el mismo. La Municipalidad no ha avanzado de manera integral y decidida en este tema. También se carece de un enfoque legal y económico que penalice en serio las conductas antisociales como tirar papeles en la calle, tirar agua de las piletas, arrojar escombros en lotes baldíos, etc.

- Si se ha avanzado en algunos procesos como el reciclado de envases de PVC, latas y vidrio con la instalación de algunos lugares puntuales para depositarlos como en el centro de la ciudad, en la misma ciudad universitaria o en determinados centros comerciales, supermercados e hipermercados, aún queda hacerlo más extensivo.

Por último, es de esperar que el cambio que se inicia en el pensamiento y la actitud sea en cierta manera impulsado por las generaciones más jóvenes, invitando a los adultos a debatir y actuar en consecuencia, para que el mismo sea realidad hoy y no mañana.

\section{BibliografíA}

Almirón, Z.; Bianchi, E. C.; Kosciuk de Gesualdo, G. y Ferreyra, S. (2011). Contribuciones al Consumo Responsable. Una mirada regional, XXV Encuentro De Docentes Universitarios de Comercialización de Argentina y América Latina, San Juan, Argentina. Disponible en: http://www.educa-al.com. ar/educa/index.php/trabajos-presentados/2011.

Alonso Rivas, J. y Grande Esteban, I. (2012). Comportamiento del consumidor. Decisiones y estrategias de marketing. Sexta edición, ESIC-Alfaomega, pp. 507. Alonso, L. B. (2006).El cambio social en España. Visiones y retos de futuro, Centro de Estudios Andaluces. Consejería de la Presidencia. 
Ballesteros, C. (2011). “Soberanía consumidora. Más allá del consumo responsable", en ¿Cambiar el Mundo desde el Consumo? Economistas sin Fronteras. Dossieres EsF. ${ }^{\circ}$ 2, julio.

Baudrillard, J. (1974). La sociedad de consumo. Sus mitos, sus estructuras. España: Plaza \& Janes, pág. 254.

Bauman, Z. (2002). Modernidad liquida. Buenos Aires: Fondo de Cultura Económica, pág. 232.

Bell, D. (1978). Las contradicciones culturales del capitalismo. Madrid: Alianza.

Bianchi, E. C. (2009). Estilos de vida en el mercado de la Tercera Edad de la ciudad de Córdoba, XXIII Encuentro De Docentes Universitarios de Comercialización de Argentina y América Latina. Córdoba, Argentina. Disponible en: http://www.educa-al.com.ar/educa/images/trabajos/10\%20-\%20 estilo\%20de\%20vida.pdf.

Bianchi, E. C. (2007). Estilo de Vida de los Jóvenes Universitarios de Córdoba, Investigación de la cátedra de Seminario de Investigación de Mercado de la Universidad Católica de Córdoba. Disponible en: http://www.ucc. edu.ar/portalucc/archivos/File/FACEA/Encuesta_universitarios/invest igacionuniversitarios.pdf.

Carrero Bosch, I.; Martínez, C. y Rosa Duran, J. (2010). La relación del consumidor con las etiquetas sociales y medioambientales Estudio diagnóstico para orientar la definición de políticas públicas y la acción empresarial, Universidad Pontifica Comillas. Madrid.

Cortes Funes, E. (2011). Consumo responsable, el rol de las empresas, del Estado y del Consumidor, Fundación Ambiente y Recursos Naturales (FARN) Informe Ambiental Anual. Argentina.

Cortina, A. (2002). Por una ética del consumo. Madrid: Taurus.

Cortina, A. y Contreras, I. (2003), "Consumo...luego existo", Cuaderno de Cristianisme i Justicia 123, Barcelona: [s.e.].

Díaz, E. (2011), "Abanico de opciones para ciudadanos responsables", en ¿Cambiar el Mundo desde el Consumo?, Economistas sin Fronteras, Dossieres EsF, $\mathrm{n}^{\mathrm{O}} 2$, julio.

Fennell, G. (1987) “Marketing, Ética y Calidad de Vida", en Samli, C. A. Marketing y la Calidad de vida, Conecticut: Quorum Books. 
Galbraith, J. K. (2005), Introducción a la economía. Una guía para todos (o casi), [s.1.]: Biblioteca de Bolsillo.

Hayek, F. (1978). Nuevos Estudios en Filosofía, Política, Economía y la Historia de las Ideas. Chicago: University of Chicago Press.

Hayek, F. (1961). Libertad bajo la ley. Disponible en .http://www.hacer. org/pdf/Hayek12.pdf

Iglesias, J. (2009). “La irresponsabilidad del consumo responsable como propuesta transformación social". Kaos en la red.

Martínez Martínez, M.; Montes del Castillo, A. y Carrillo Escobar, M. (2008). Comercio Justo y Consumo Responsable en la Universidad de Murcia. Nuevas propuestas, Anales de IV Congreso Universidad y Cooperación al Desarrollo. Barcelona.

Martinez, Juan A. F. (2008). La teología del Mercado, VI Jornadas de Economía Crítica, Bilbao, 27 al 29 de marzo.

Martínez, Pepe (2008). Cualitativa-mente. Los secretos de la investigación cualitativa. España: ESIC Editorial, pág. 341.

Micheletti, M. (2003).Political virtue and shopping: Individuals, consumerism, and collective action. Nueva York: Palgrave Macmillan.

Miller, Daniel (1999). Ir de compras: una teoría. México: Siglo XXI.

Moltedo Perfetti, P. (2007). "Reflexiones en torno al Marketing y la Felicidad", en Loreto Marchant, R. Actualizaciones para el Management y el Desarrollo Organizacional, Universidad del Viña del Mar. Disponible en: www.eumed.net/libros/2007a/223/pmp.htm.

Reisch, L. (2011). "Prólogo", en RodríguezVillarino, R. y Otero-López, J. M. Adicción a la compra. Análisis, evaluación y tratamiento. Madrid: Pirámide.

Salcedo Aznal, A. y Garces Prieto, J. (2006). “Influencia de la ansiedad en la adicción al consumo y la falta de autocontrol en la compra y el gasto de los jóvenes", Estudios sobre Consumo, volu. 76, España.

Santesmases Mestre, M.; Sánchez de Dusso, F.y Kosciuk de Gesualdo, G. (2004). Marketing, conceptos y estrategias, $2^{\circ}$ edición, Buenos Aires: Pirámide.

Szmigin, I.; Carrigan, M. y McEachern, M. (2009). The conscious consumer: taking a flexible approach to ethical behaviour, forthcoming, in the special issue of International Journal of Consumer Studies on Sustainable Consumption. 
Anexo I-Batería de ítems de Consumo Responsable

Ítems de Consumo Responsable

1. Siempre leo la fecha de vencimiento cuando compro alimentos.

2. Acostumbro comprar los mismos productos que compran mis amigos.

3. Me informo de las ofertas y promociones disponibles para hacer una compra más conveniente.

4. Una marca con prestigio respalda la calidad de los productos.

5. Antes de comprar un producto comparo precios.

6. Valoro si el producto es útil o necesario antes de efectuar una compra.

7. No conozco las entidades en las que puedo reclamar mis derechos como consumidor.

8. Los grandes almacenes e hipermercados me agobian.

9. Ante 2 productos similares, compraría el de una empresa socialmente responsable aunque tenga que pagar un poco más.

10. Los avisos publicitarios brindan información suficiente para la compra de productos.

11. Prefiero salir sola/o de compras porque tengo libertad para elegir todo lo que quiero.

12. Cuando compro indumentaria, la marca del producto es determinante.

13. Ver tiendas es una de las cosas divertidas que se pueden hacer en una ciudad.

14. El Estado debe tener un rol activo para apoyar e incentivar la actuación responsable de empresas y consumidores.

15. Los consumidores pueden influir para que las empresas tengan un comportamiento socialmente responsable.

16. Las empresas hacen actividades de RSE (ej. capacitación de empleados, donaciones, cuidado del medio ambiente, etc.) solo para vender más.

17. Muchas cosas las compramos pensando en dar envidia a los demás.

18. Siempre guardo algo de dinero para poder hacer frente a los gastos imprevistos.

19. Me gusta pasar el tiempo en los grandes centros comerciales.

20. Tener dinero y comprar cosas caras como buenos coches o ropa de marca, me sirven para aumentar mi condición social.

21. Cuando más compro y consumo, más feliz soy.

22. Cuando recibo información de conductas no éticas de empresas, me encargo de difundirlas.

23. Me molesta que las empresas contaminen el medio ambiente.

24. Muchas veces compro cosas inútiles que después me arrepiento de haber comprado.

25. Elijo productos con packaging ecológico o reciclable.

26. Siento la necesidad de salir a comprar.

27. Leo los prospectos y la información de los envases de los productos antes de comprar.

28. En mi casa separamos los residuos sólidos (botellas, papel, plástico, etc.) del resto.

29. En general, hago una ducha corta cuidando el agua.

30. Tengo la costumbre de reclamar por la factura de compra cuando no me la dan.

31. Apago las luces y aprovecho al máximo las horas de luz solar.

32. Me interesa conocer acerca del comportamiento ético de las empresas.

33. Cuando en una tienda algo que me gusta, no me lo puedo quitar de la cabeza hasta que lo compro.

34. Cuando estoy en una localidad pequeña extraño los shoppings y las tiendas de las ciudades.

35. Desconozco las políticas ambientales de las empresas a las cuales compro productos.

36. Reutilizo papel en la impresión de documentos.

37. Siempre guardo el ticket de compra por cualquier reclamo que tenga que efectuar.

38. En general, planifico la compra de alimentos.

39. Tengo la costumbre de comprar alguna ropa o calzado todos los meses.

40. No conozco/no se cuales son los llamados 'productos orgánicos'.

41. Compro productos que no son originales porque me resultan más barato.

42. Creo que las nuevas generaciones tienen más conciencia sobre el cuidado del medio ambiente.

43. El Estado no realiza ninguna acción para promover el consumo responsable.

44. Cambio el teléfono celular por uno nuevo por lo menos una vez al año.

45. Compro las cosas que me gustan, sin tener en cuenta si son ecológicas o no.

46. Compro ropa solo cuando necesito, no me importa estar a la moda.

47. Se puede ser una persona consumista y a la vez consumir de manera responsable. 
Anexo II - Baterías de ítems para Estilos de Vida ${ }^{6}$

Baterías de ítems para medir los Estilos de Vida Tiempo Libre/ Recreación/Medios de Comunicación

1. Me gusta más hacer deportes grupales o en equipos (fútbol, básquet, etc.).

2. Tomarme vacaciones fuera de mi casa es muy importante.

3. Voy a un gimnasio habitualmente porque mejora mi calidad de vida.

4. Cuando estoy cansado/a de estudiar generalmente miro TV o visito amigos.

5. La mayoría de los estudiantes hace menos gimnasia de lo que deberían.

6. Leo diarios para informarme en detalle de la actualidad político-social.

7. La TV e Internet remplazaron la lectura de libros, diarios y revistas.

8. Compro revistas de actualidad - políticas, económicas o especializadas - para mantenerme informado.

9. Escucho radio FM solo por la música.

10. Leer por placer me resulta poco atractivo.

11. No compro libros, diarios ni revistas porque no me alcanza el dinero.

12. La información que recibo de los medios de comunicación es poco confiable.

Salud/ Alimentación

1. Estar en forma no es sinónimo de estar saludable.

2. Trato de consumir productos sanos como frutas y verduras.

3. Comer sano es más costoso.

4. Mi alimentación no es adecuada debido a mi rutina diaria.

5. Consumir productos light ayuda a lograr mi peso ideal.

6. La carne vacuna es una parte importante de mi alimentación.

7. La comida rápida es perjudicial para el cuidado del cuerpo.

8. El consumo de productos light es una moda.

9. A veces tomo medicamentos por la recomendación de un amigo/a.

10. Las dietas estrictas son la única opción para bajar de peso.

11. La actividad física es fundamental para estar saludable.

12. Voy al médico solo cuando me duele algo.

13. Nunca asistiría a un hospital público.

\section{Actividad Física}

1. Considero que no es necesario realizar actividades físicas para lograr una buena imagen.

2. El yoga, pilates y fitness ayudan más que otras disciplinas a moldear un cuerpo ideal.

3. Práctico deportes por hobby.

4. Los deportes grupales permiten una mejor sociabilización.26. ActFisL1. Considero que no es necesario realizar actividades físicas para lograr una buena imagen.

Belleza

$1 . \quad$ Siempre que tengo tiempo realizo algún tratamiento de belleza o estética.

2. Me agrada que los hombres dediquen un poco más de su tiempo al cuidado de su estética personal

3. Un buen corte de cabello realza mi imagen.

4. Perfumarse y oler bien me da seguridad a la hora de relacionarme y comunicarme con los demás.

5. No tiene sentido usar cosméticos para la piel que rejuvenezcan mi cuerpo .

6. Evito mirar las revistas pues lo único que muestran son modelos delgadas y siempre impecables.

7. Me preocupa cómo combatir la celulitis. (mujeres).

8. Son entendibles las cirugías estéticas solo por algún problema de salud.

9. Si no te sentís bien con tu cuerpo es coherente realizarse cirugías estéticas para verse bien

10. Si tuviese el dinero suficiente me realizaría una cirugía plástica.

11. La mejor forma de bajar de peso es la liposucción o el cinturón gástrico.

12. Los hombres le temen al quirófano, solo enfrentan las cirugías por necesidad.

13. La cirugía para la obesidad es más frecuente en los hombres que en las mujeres.

${ }^{6}$ Escala de Likert de 5 puntos: TA -PA-NI -PD-TD. 


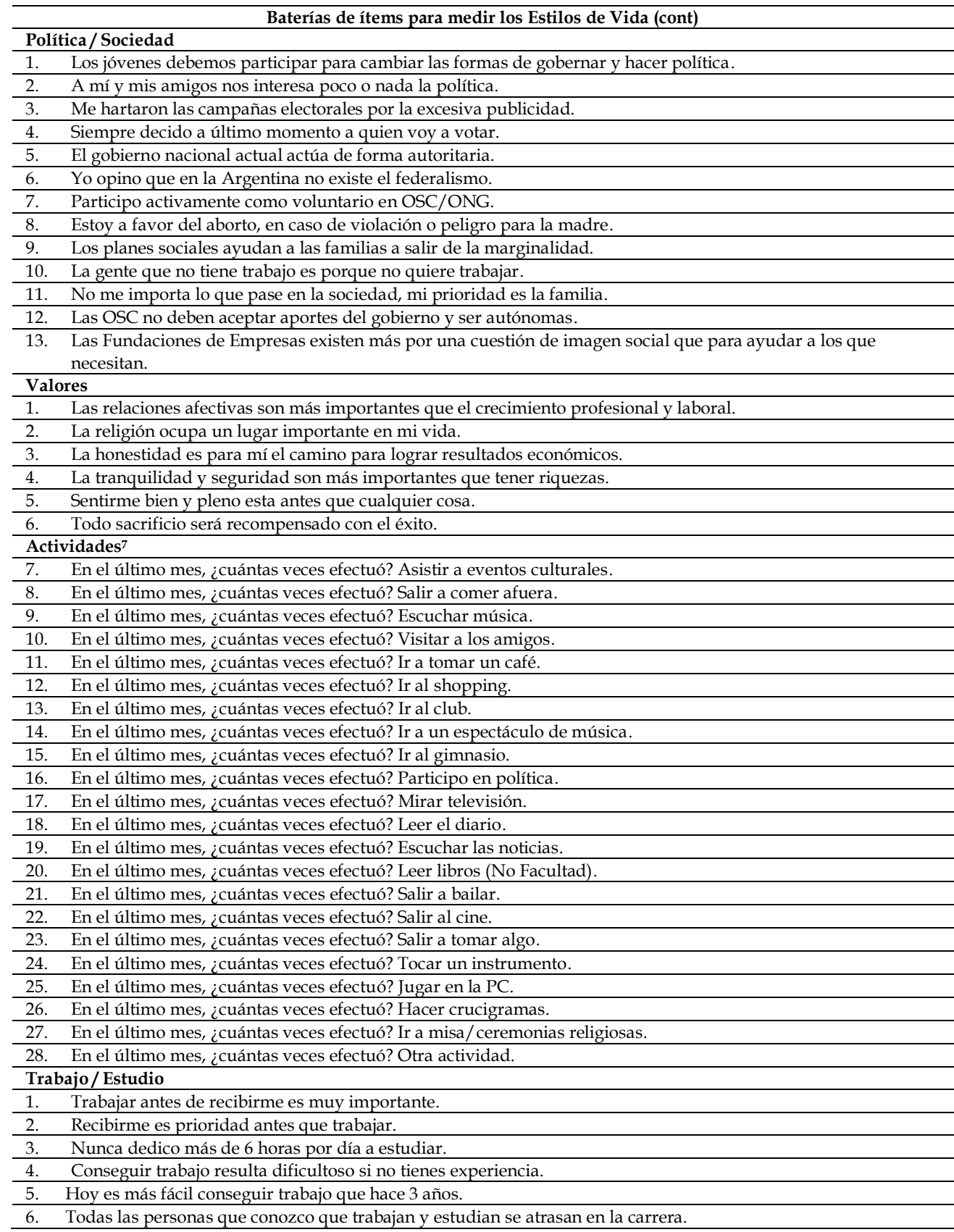

${ }^{7}$ Escala: Más de 2 veces por semana -1 a 2 veces por semana - 2 o 3 veces por mes -1 vez por mes - No efectúo. 
Anexo III - Tipología de consumidores responsables

\begin{tabular}{|c|c|c|}
\hline Tipología & Características / Descripción & Peso \\
\hline $\begin{array}{l}\text { Indiferentes/ } \\
\text { Apáticos }\end{array}$ & $\begin{array}{l}\text { Este grupo de consumidores son los que menos guardan los tickets de compras, y } \\
\text { manifiestan no leer los vencimientos de los productos. No planifican las compras y no } \\
\text { están al tanto de las ofertas y promociones previamente a la compra. } \\
\text { En cuanto al comportamiento, no han incorporado conductas responsables pues no } \\
\text { reutilizan papel en la impresión de documentos, son los que menos dicen apagar las } \\
\text { luces y aprovechar la luz natural entre otras. }\end{array}$ & $20 \%$ \\
\hline $\begin{array}{l}\text { Anticonsumistas/ } \\
\text { Conscientes }\end{array}$ & $\begin{array}{l}\text { A este grupo de consumidores no los hace feliz el consumo. Respecto del lugar de la } \\
\text { compra, les agobian los hiper y super, y a la hora de salir de comprar prefieren salir } \\
\text { solos/as. Se sienten muy seguros de lo que buscan, no se guían por las compras de } \\
\text { sus amigas/os. No sienten la ansiedad o necesidad de salir a comprar. Encuentran en } \\
\text { parte refugio en las marcas, pues para ellos una marca de prestigio respalda la calidad } \\
\text { del producto. Este grupo no tienen una postura firme frente a conductas como elegir } \\
\text { productos de pack ecológicos, separar residuos, cuidar el agua. Son los que más } \\
\text { guardan los tickets. }\end{array}$ & $24 \%$ \\
\hline $\begin{array}{l}\text { Consumidores } \\
\text { Responsables }\end{array}$ & $\begin{array}{l}\text { Son los que más se preocupan por elegir productos de pack ecológicos, separar } \\
\text { residuos, cuidar el agua en la ducha. Aunque se interesan parcialmente en conocer el } \\
\text { comportamiento ético de las empresas, son los que se encargan de difundirlas cuando } \\
\text { conocen de casos no éticos. Se preocupan por conocer las políticas ambientales de las } \\
\text { empresas. Sin embargo, a pesar de ser conscientes tiene en claro que son consumistas: } \\
\text { dicen sentirse en gran parte más feliz cuando más compran y consumen, extrañan los } \\
\text { shopping si están fuera de la ciudad, y sienten fuertemente la necesidad de comprar. } \\
\text { Por lo anteriormente descripto, es el grupo que más se aproxima a las definiciones } \\
\text { conceptuales analizadas sobre que es un consumidor responsable. }\end{array}$ & $31 \%$ \\
\hline $\begin{array}{l}\text { Shopper/ } \\
\text { Compulsivos }\end{array}$ & $\begin{array}{l}\text { A este grupo le encanta ver tiendas en la ciudad, le gustan los centros comerciales, } \\
\text { compran todos los meses alguna ropa o calzado, cuando ven algo no se lo pueden } \\
\text { sacar de la cabeza. Las marcas son en parte determinantes a la hora de comprar } \\
\text { indumentaria. } \\
\text { Lo que lo caracteriza es su baja implicación en la compra desde el punto de vista } \\
\text { racional y ético, pues les interesa poco el comportamiento de las empresas y no } \\
\text { difunden los comportamientos negativos de estas. Ante un caso de } 2 \text { productos } \\
\text { similares, no pagarían de más por ser de una Empresa Socialmente Responsable } \\
\text { (RSE). Son los que menos conocen las entidades de defensa del consumidor. Su nivel } \\
\text { de impulsividad en la compra hacen que sean los/las que más se arrepienten de las } \\
\text { compras inútiles que hacen. Debido a su bajo nivel de consciencia social respecto del } \\
\text { impacto del consumo hace que no eligen productos ecológicos, ni separan residuos en } \\
\text { sus casas, ni ahorran agua en la ducha. }\end{array}$ & $25 \%$ \\
\hline
\end{tabular}

Fuente: Almirón, Z, Bianchi, E; Ferreyra, S y Gesualdo, G. (2011). Contribuciones al Consumo Respon-sable, una mirada regional. XXIV Educa-AL. San Juan. Argentina. setiembre. 